\title{
Prediction of a Francis Turbine Prototype Full Load Instability From Investigations on the Reduced Scale Model
}

\author{
Sébastien Alligné ${ }^{1}$, Pierre Maruzewski ${ }^{1}$, Timothy Dinh ${ }^{2}$, Ben Wang ${ }^{2}$, Andrei Fedorov ${ }^{2}$, Jacob Iosfin ${ }^{2}$ \\ and François Avellan ${ }^{1}$ \\ ${ }^{1}$ EPFL Laboratory for Hydraulic Machines, \\ Av. de Cour 33 bis, Lausanne, 1007, Switzerland, sebastien.alligne@epfl.ch, pierre.maruzewski@epfl.ch, \\ françois.avellan@epfl.ch \\ ${ }^{2}$ BC Hydro Generation Engineering - Turbines \\ 6911 Southpoint Dr, Burnaby, Canada,timothy.dinh@bchydro.com, ben.wang@bchydro.com, \\ andrei.fedorov@bchydro.com, jacob.iosfin@bchydro.com
}

\begin{abstract}
The growing development of renewable energies combined with the process of privatization, lead to a change of economical energy market strategies. Instantaneous pricings of electricity as a function of demand or predictions, induces profitable peak productions which are mainly covered by hydroelectric power plants. Therefore, operators harness more hydroelectric facilities at full load operating conditions. However, the Francis Turbine features an axisymmetric rope leaving the runner which may act under certain conditions as an internal energy source leading to instability. Undesired power and pressure fluctuations are induced which may limit the maximum available power output. BC Hydro experiences such constraints in a hydroelectric power plant consisting of four 435 MW Francis Turbine generating units, which is located in Canada's province of British Columbia. Under specific full load operating conditions, one unit experiences power and pressure fluctuations at $0.46 \mathrm{~Hz}$.

The aim of the paper is to present a methodology allowing prediction of this prototype's instability frequency from investigations on the reduced scale model. A new hydro acoustic vortex rope model has been developed in SIMSEN software, taking into account the energy dissipation due to the thermodynamic exchange between the gas and the surrounding liquid. A combination of measurements, CFD simulations and computation of eigenmodes of the reduced scale model installed on test rig, allows the accurate calibration of the vortex rope model parameters at the model scale. Then, transposition of parameters to the prototype according to similitude laws is applied and stability analysis of the power plant is performed. The eigenfrequency of $0.39 \mathrm{~Hz}$ related to the first eigenmode of the power plant is determined to be unstable. Predicted frequency of the full load power and pressure fluctuations at the unit unstable operating point is found to be in general agreement with the prototype measurements.
\end{abstract}

Keywords: Francis Turbine, Full load instability, Transposition, Hydro acoustic

\section{Introduction}

In full load operation, Francis Turbines feature an axi-symmetric rope leaving the runner which may act under certain conditions as an internal energy source leading to instability. Undesired power and pressure fluctuations are induced which may limit the maximum available power output. In 1988, Jacob et al. [1] performed the first experimental investigations on the full load instability phenomenon. They analyzed pressure fluctuations on the reduced scale model of a prototype which featured this instability. Unfortunately the surge instability on the test rig was much less significant in comparison to the prototype. However they pointed out that the pressure fluctuations frequency corresponds to one of the eigenfrequencies of the hydraulic system which they computed from a model based on the cavitation compliance parameter determined by experimental measurements. The same methodology was used to explain high load pressure fluctuations occuring in a two 140MW Francis Turbine unit power plant in 1992 [2]. Furthermore, modelling of the full load vortex rope was based on a key parameter called the mass flow gain factor defined as the rate of change of the vortex rope cavity volume with respect to change of the discharge. Initially this parameter was introduced in combination with the cavitation compliance by Brennen and Acosta in 1976 [3] and 1978 [4] to analyze the POGO instability experienced by pumps in liquid propellant rockets. Koutnik and Pulpitel [5] applied this modelling approach to Francis 
Turbines. Using the transfer matrix method, they derived a stability diagram to explain a full load surge occurring on a four 39MW Francis Turbine unit power plant. A similar approach based on mapping cavitation parameters was also successfully applied to explain inducer instabilities by Tsujimoto et al. in 1993 [6] and propeller instabilities by Duttweiler and Brennen in 2002 [7] and by Watanabe and Brennen in 2003 [8]. The mass flow gain factor used by Koutnik and Pulpitel is defined according to the discharge downstream of the cavity volume. However, Chen et al. [9] derived a more complicated definition from the velocity triangle at the runner outlet which takes into account the discharge both upstream and downstream of the cavity. With an analytical model they analyzed the influence of the swirl intensity and the diffuser factor on the system stability. More recently, deriving the cavitation compliance and the mass flow gain factor parameters from CFD simulations of the complete machine, Flemming et al [10] predicted instability of a prototype installation based on eigenvalues and eigenmodes analysis. However, validation was not available. In 2006, Koutnik et al. [11] performed a time domain simulation of a self oscillation occurring in a four 400MW Francis Pumped-Storage power plant. In order to obtain finite fluctuations called "limit cycle", nonlinearity of the cavitation compliance as a function of the Thoma number is introduced. Moreover, the system is pushed to the limit of instability by setting a mass flow gain factor parameter very low compared to the one derived from the stability analysis. Therefore, during the transient, the system enters unstable conditions and nonlinearity of the compliance switches between stable and unstable conditions allowing the system to reach the limit cycle. It has been shown by authors that the limit cycle is reachable only if nonlinearity of cavitation compliance is taken into account. In 2009, Alligné et al. [12] derived a new model for the full load vortex rope taking into account an additional dissipation due to the thermodynamic exchange between the gas and the surrounding liquid. Therefore, three key hydro acoustic parameters must be set-up: the cavitation compliance or the wave speed, the mass flow gain factor and the additional dissipation called viscoelastic damping. It has been confirmed that nonlinearity of the parameters is necessary to reach the limit cycle, especially for the viscoelastic damping.

The BC Hydro’s Mica hydroelectric power plant, located in Canada’s province of British Columbia, experiences the full load instability phenomenon when unit operates at power outputs in excess of 460MW. This paper presents the methodology developed to predict the instability on the Mica power plant prototype using both experimental measurements and CFD simulations performed on the reduced scale model. The full load vortex rope model including the viscoelastic damping parameter is used and calibrated for the reduced scale model. Then, transposition laws for the hydro acoustic model parameters are established and stability of the prototype is assessed by modal analysis.

\section{Prototype instability}

Commissioned in 1970's, the Mica powerhouse features four existing 435MW generating units, each equipped with a Francis type turbine rated at power output of $443.7 \mathrm{MW}$ under the $170.7 \mathrm{~m}$ rated net head, . Two additional units are planned to be installed at Mica power plant in the future. Each existing unit is supplied by individual power conduits including intake, penstock and draft tube. Units 1, 2 and 3 share a common tailrace tunnel (i.e. free surface flow) while Unit 4 and two future units share another common tailrace tunnel. The length of each penstock is about 310 meters and the tailrace tunnel each is about 425 meters long. Pressure and power fluctuations are experienced when three out of four Mica units are operated simultaneously. The surging scenario corresponds to Unit 1 at full load; Unit 4 shutdown and both Unit 2 and Unit 3 operated near their best efficiency points. Under these conditions, Unit 1 was the only unit which experienced high power swings and high pressure fluctuations in the penstock and draft tube. Site measurements were performed to provided automatic data acquisition to record the surging. The measured frequency of Mica Unit 1 full load instability was $0.46 \mathrm{~Hz}$. The aim of this paper is to predict the instability at this frequency from investigations on the reduced scale model.

\section{Measurements on reduced scale model}

The reduced scale model of the Mica prototype turbine for units 1 and 2 was installed and tested on the EPFL PF2 test rig of the Laboratory for Hydraulic Machines. Performance tests were carried out according to the IEC 60193 standard [13]. Dynamic pressure sensors were installed in the draft tube and synchronized with a high speed video camera to allow visualization of the behaviour of the cavity volume of the vortex rope in the cone. The investigation on the reduced scale model is carried out at the Froude similitude to overcome scale effects on the vortex rope cavity volume regarding transposition from the reduced scale model to the prototype. Indeed, this non-dimensional number affects the distribution of cavitation in the flow as it determines the pressure gradient relative to the size of the machine [14]. This condition allows defining the specific energy $E$ of the reduced scale model. Rotational speed is imposed by the equality of the speed factor $N_{E D}$ between the reduced scale model and prototype, and the guide vane opening $y$ is setup to correspond to the prototype power output. Finally, the Thoma number is derived based on the machine setting level of the power plant. The measurement conditions on the reduced scale model installed on test rig are summarized in Tab. 1.

Table 1 Investigated operating point

\begin{tabular}{ccccccc}
\hline $\begin{array}{c}\mathrm{E} \\
{\left[\mathrm{J}_{\mathrm{kg}}{ }^{-1}\right]}\end{array}$ & $\begin{array}{c}\mathrm{Q} \\
{\left[\mathrm{m}^{3} \cdot \mathrm{s}^{-1}\right]}\end{array}$ & $\begin{array}{c}\mathrm{N}_{\mathrm{ED}} \\
{[-]}\end{array}$ & $\begin{array}{c}\mathrm{QED}_{\mathrm{ED}} \\
{[-]}\end{array}$ & $\begin{array}{c}\mathrm{T}_{\mathrm{ED}} \\
{[-]}\end{array}$ & $\begin{array}{c}\mathrm{y} \\
{\left[{ }^{\circ}\right]}\end{array}$ & $\begin{array}{c}\sigma \\
{[-]}\end{array}$ \\
\hline 115.8 & 0.347 & 0.273 & 0.263 & 0.133 & 30 & 0.11 \\
\hline
\end{tabular}


Under these conditions, the cavity volume experienced pulsations by intermittence which yields the conclusion that this operating point is at the limit of instability on the test rig. When instability occurs, the vortex rope cavity volume fluctuates as shown in Fig. 1 whereas under stable conditions, the vortex rope cavity volume is constant.

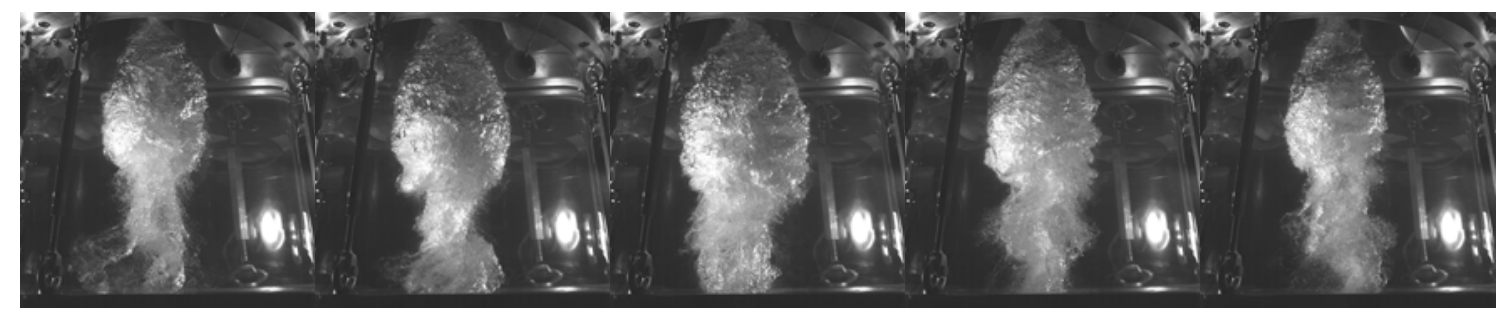

Fig.1 Vortex rope cavity volume pulsations

The intermittent pulsation is detected with the dynamic pressure sensors located in the cone. Time history of the pressure coefficient $c_{p}$ defined by Eq. 1 and frequency spectra of pressure fluctuations $\tilde{p}$ of one pressure sensor are given respectively in Fig.2 a) and Fig.2 b).

$$
c_{p}=\frac{\tilde{p}}{\frac{1}{2} \cdot \rho \cdot U_{1}^{2}}
$$

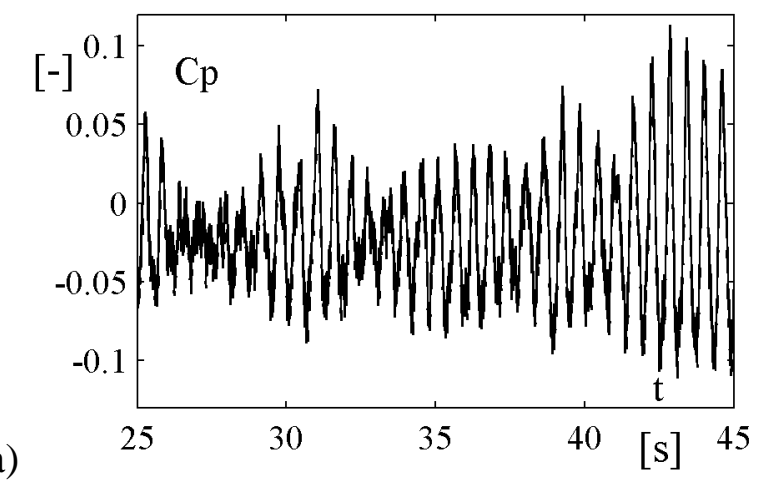

Fig.2 a) Time history and b) frequency spectra of pressure fluctuations in the cone

The fundamental instability frequency of pressure signals is $f=1.6 \mathrm{~Hz}$ which corresponds to the vortex rope pulsation observed with the high speed camera. Moreover, a high frequency of $f=3.5 \mathrm{~Hz}$ which is not the harmonic of the fundamental, is exhibited in the pressure signal. This frequency is dominant when the vortex rope is stable and does not experience pulsations. In the time history, this stable phase is located for a low pressure fluctuations level as observed in Fig. 2 a) at time $t=27 \mathrm{~s}$. This high frequency is assumed to be due to the local 3D flow in the cone whereas the low frequency is one of the eigenfrequency of the system.

\section{Hydro-acoustic model}

The test rig and the investigated reduced scale model have been implemented in the one dimensional hydro-acoustic SIMSEN software as it has been shown in previous work [12]. Due to the onset of the vortex rope cavity volume, the cone and the elbow parts of the draft tube between sections $\overline{1}$ and $\overline{2}$ are modelled by the equivalent electrical scheme given in Fig. 3 [12].

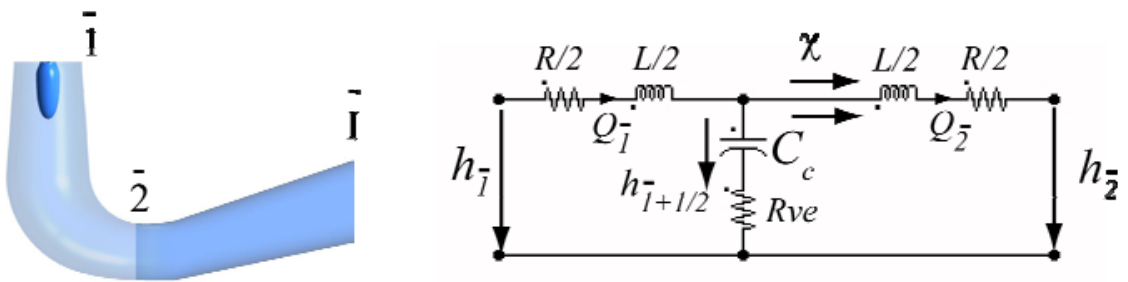

Fig.3 Draft tube domain (left) and equivalent electrical scheme of the set of cone and elbow parts (right) 
In the framework of this investigation, one pressure node or capacitance is used to model the vortex rope. The corresponding continuity equation is then:

$$
Q_{\overline{1}}-Q_{\overline{2}}=\frac{D V_{c}}{D t}=C_{c} \frac{d h_{\overline{1}+1 / 2}}{d t}+\chi \frac{d Q_{\overline{2}}}{d t}
$$

with $C_{c}$ and $\chi$ respectively the cavitation compliance and the mass flow gain factor defined as:

$$
C_{c}=-\frac{\partial V_{c}}{\partial h_{\overline{1}+1 / 2}}=\frac{g \cdot A \cdot l}{a^{2}} \quad \chi=-\frac{\partial V_{c}}{\partial Q_{\overline{2}}}
$$

Three key hydro-acoustic parameters must be set up to model the full load vortex rope:

- $\quad$ the mass flow gain factor which represents the excitation mass source of the hydraulic system;

- $\quad$ the wave speed which defines the cavitation compliance;

- $\quad$ the equivalent viscoelastic damping defining the resistance in series with the capacitance which models thermodynamic exchange between liquid and gas [15].

\section{CFD computation of the mass flow gain factor parameter}

CFD simulations of the three dimensional flow in the draft tube, with the commercial program ANSYS CFX 11.0 are performed to compute the mass flow gain factor parameter which is the excitation mass source of the system. This software, based on finite volume methods, solves the incompressible steady Reynolds Average Navier-Stokes equations in their conservative form. The set of equations is closed with a two-equation turbulence model, the Shear Stress Transport, see Menter [16]. Moreover, the "High resolution" advection scheme is used. The computational domain is the complete machine consisting of the spiral case, the stay vanes, the guide vanes, the runner and the draft tube. The rotating part containing the runner features two interfaces "Frozen Rotor" with the stationary parts such as the guide vane row and the draft tube. The computing domain is discretized with a structured mesh of 6 million nodes carried out with ANSYS ICEM 11.0. The boundary conditions are a prescribed discharge at the spiral case inlet and an opening condition with "static pressure for entrainment" at the draft tube outlet. Single phase simulations are considered for all the flow numerical simulations. Hence, to derive the hydro-acoustic parameters, the vortex rope cavity volume is assumed to correspond to the flow region bounded by the vapour pressure $p_{v}$ set to $2338 \mathrm{~Pa}$ at $20^{\circ} \mathrm{C}$.

The mass flow gain factor is defined as the vortex rope cavity volume variation as function of discharge variation, see Eq. 3 . Therefore, to derive this parameter corresponding to the investigated operating point, three flow numerical simulations are performed. For each simulation, the same outlet pressure derived from the Thoma number of the experiment is prescribed. However, three inlet discharges are specified: the nominal discharge $Q_{n}$ of the experiment compared to a higher and lower discharge difference of 5\%, see Fig. 4.
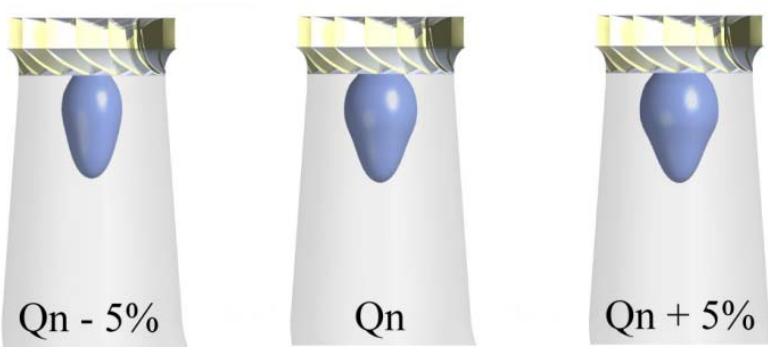

Fig.4 Variation of the vortex rope volume as function of the discharge

The central finite difference approximation leads to a mass flow gain factor value of $\chi=-0.039 \mathrm{~s}$ at the experimental discharge $Q_{n}$.

\section{Computation of the wave speed and the viscoelastic damping}

Using a small perturbation stability analysis of the reduced scale model installed on the test rig, the wave speed and the viscoelastic damping in the draft tube are computed. This stability analysis in SIMSEN is based on the linearization of the set of hydro acoustic differential equations of the system around the operating point and then, stability is deduced from eigenvalues of the linearized set of equations [17]. Damping and oscillation frequency of the eigenmodes are given respectively by the real part and the imaginary part of the eigenvalues.

To derive the wave speed corresponding to the investigated operating point, eigenfrequencies of the test rig are computed for different wave speeds in the draft tube, see Fig. 5. 


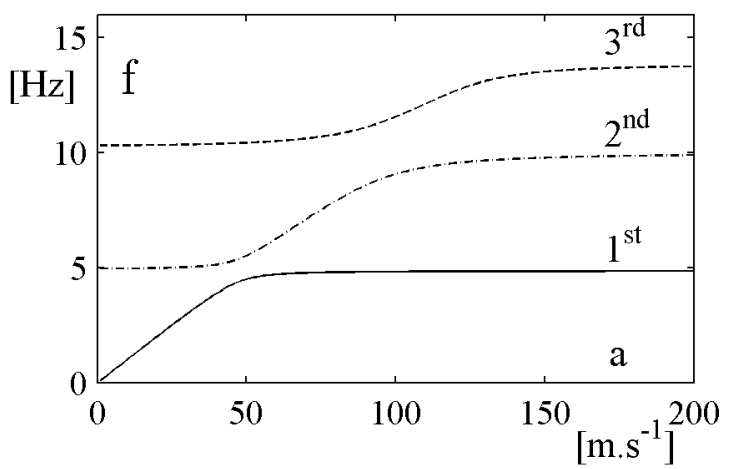

Fig.5 Eigenfrequencies as function of the wave speed in the draft tube.

For a critical wave speed value depending on the eigenmode considered, the eigenfrequency decreases when the wave speed is decreased. To determine the wave speed induced by the cavity volume of the vortex rope, the experimental unstable frequency of $f_{\exp }=1.6 \mathrm{~Hz}$ is used. Since this unstable frequency corresponds to an eigenfrequency of the system [1,12], this frequency is reached by the first eigenmode when the wave speed is set to $a=15 \mathrm{~m} . \mathrm{s}^{-1}$.

The computation of the viscoelastic damping of the vortex rope model is based on the stability diagram of the system. For a given viscoelastic damping, eigenvalues are computed for pairs of parameters $a-\chi$. If at least one of the eigenvalues has a positive real part, the system is identified as unstable. In Fig. 6, two stability diagrams are computed for two viscoelastic dampings. Moreover the set of parameters $a-\chi$ derived respectively from eigenfrequencies computation and CFD simulations are located in the diagram.

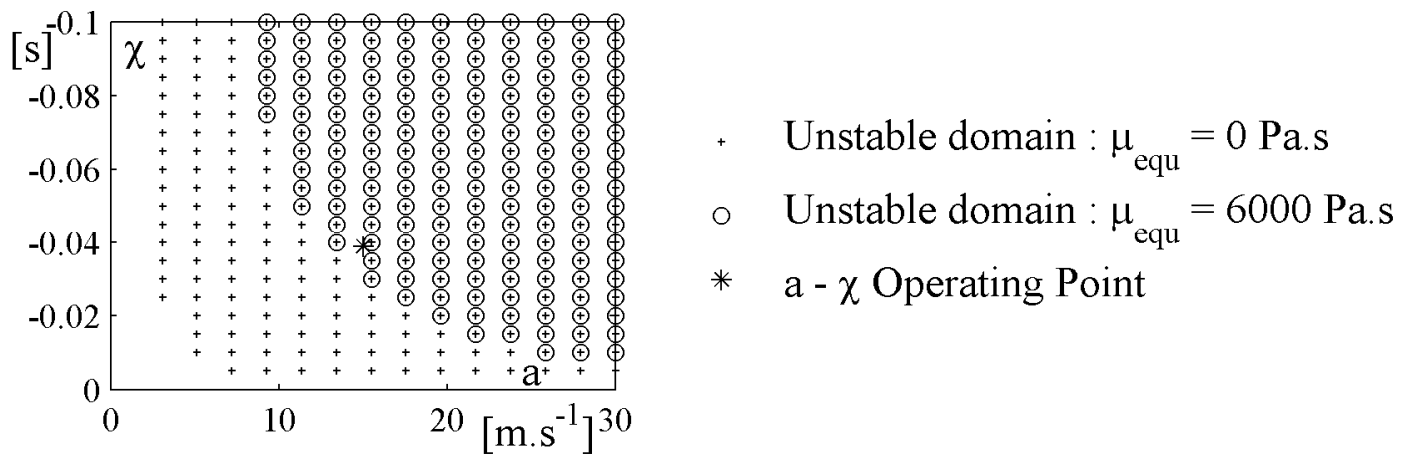

Fig.6 Stability diagrams.

By modifying the viscoelastic damping, the limit of stability moves accordingly. The more the damping is increased, the more the system is stable. In Section 3, experiments showed that the system is at the limit of stability. Therefore, the viscoelastic damping is set to $\mu_{\text {equ }}=6000$ Pa.s to move the stability limit up to the computed set of parameters $a-\chi$, see Tab. 2 .

Table 2 Reduced scale model parameters

\begin{tabular}{ccc}
\hline \hline $\begin{array}{c}\mathrm{a} \\
{\left[\mathrm{m} . \mathrm{s}^{-1}\right]}\end{array}$ & $\begin{array}{c}\chi \\
{[\mathrm{s}]}\end{array}$ & $\begin{array}{c}\mu \\
{[\text { Pa.s }]}\end{array}$ \\
\hline 15 & -0.039 & 6000 \\
\hline
\end{tabular}

\section{Transposition to the prototype draft tube}

In Sections 5 and 6, the key hydro acoustic parameters of the reduced scale draft tube have been computed with the help of both CFD numerical simulations of the three dimensional flow in the draft tube and the stability analysis of the system based on eigenfrequencies and eigenmodes computation. To investigate the stability operation of the prototype, these hydro acoustic parameters need to be transposed to the prototype conditions according to similitude laws developed in this section. To define these similitude laws, a dimensional analysis of each parameter is performed and is expressed as a function of the reference diameter $D_{\text {ref }}$ and the rotation frequency $n$ by assuming that the influence of the Reynolds number on the transposition is negligible. Then, the dimensionless parameters are defined to establish the relation between the prototype and the reduced scale model parameters. Transpositions of the wave speed and the viscoelastic damping are performed respectively through the electrical components such as capacitance or the compliance and the viscoelastic resistance. 
By definition, the compliance is proportional to the partial derivative of the cavity volume with respect to the pressure:

$$
C_{c} \sim \frac{\partial V_{c}}{\partial h}\left[\mathrm{~m}^{2}\right]
$$

Dividing by the gravity and expressing the dimensions of the cavity volume $V_{c}$ and the energy $g h$ as function of the reference diameter and the rotation frequency, it yields to:

$$
\frac{C_{c}}{g} \sim \frac{D_{r e f}^{3}}{n^{2} D_{r e f}^{2}} \sim \frac{D_{r e f}}{n^{2}}
$$

Hence, the transposition relation between the prototype and the reduced scale model compliances is:

$$
C_{c}^{P}=C_{c}^{M} \cdot\left(\frac{n^{M}}{n^{P}}\right)^{2} \cdot \frac{D_{r e f}^{P}}{D_{r e f}^{M}}
$$

where the superscripts $P$ and $M$ represent the prototype and the model values respectively.

The mass flow gain factor is proportional to the partial derivative of the vortex rope cavity volume with respect to the discharge:

$$
\chi \sim \frac{\partial V_{c}}{\partial Q} \quad[\mathrm{~s}]
$$

Expressing the dimensions of the cavity volume $V_{c}$ and the discharge as function of the reference diameter and the rotation frequency, it yields to:

$$
\chi \sim \frac{D_{r e f}^{3}}{D_{r e f}^{3} n} \sim \frac{1}{n}
$$

Hence, the transposition relation between the prototype and the reduced scale model mass flow gain factor is:

$$
\chi^{P}=\chi^{M} \cdot \frac{n^{M}}{n^{P}}
$$

The dimension of the viscoelastic resistance $R_{v e}=\mu / \rho g A l$ is $\left[\mathrm{s} . \mathrm{m}^{-2}\right]$. Since the dimensions of the mass flow gain factor and the compliance are respectively $[\mathrm{s}]$ and $\left[\mathrm{m}^{2}\right]$, the non-dimensional formulations described above with the reference diameter and the rotation frequency can be used:

$$
R_{v e} \cdot g \sim \frac{1}{n} \cdot \frac{n^{2}}{D_{r e f}}
$$

Therefore, the transposition relation between the prototype and the reduced scale model is:

$$
R_{v e}^{P}=R_{v e}^{M} \cdot \frac{D_{r e f}^{M}}{D_{r e f}^{P}} \cdot \frac{n^{P}}{n^{M}}
$$

By assuming that both the Thoma similitude and the Froude similitude conditions are fulfilled between the reduced scale model and the prototype, these transposition relations are applied to the set of parameters computed in Sections 5 and 6, see Tab. 3.

Table 3 Transposition of hydro acoustic parameters

\begin{tabular}{cccc}
\hline & $\begin{array}{c}\mathrm{a} \\
{\left[\mathrm{m} . \mathrm{s}^{-1}\right]}\end{array}$ & $\begin{array}{c}\chi \\
{[\mathrm{s}]}\end{array}$ & $\begin{array}{c}\mu \\
{[\text { Pa.s }]}\end{array}$ \\
\hline model & 15 & -0.039 & 6000 \\
prototype & 59 & -0.157 & 363707 \\
\hline
\end{tabular}

\section{Prediction of the prototype instability}

To investigate the prototype instability, the hydro acoustic SIMSEN model of the complete power plant has been setup. In the SIMSEN model, a common constant pressure at the draft tube outlets is applied. Therefore the model behaves as if each unit was isolated without any interaction between units with the same boundary conditions. This independence of the units is clearly debatable since the tailrace tunnel may induce interactions between the Units which would change independently the outlet conditions of the units and therefore would change the hydro acoustic parameters. However, for the investigated surging scenario only Unit 1 is operating at full load conditions whereas Unit 2 and Unit 3 are near their best efficiency points and Unit 4 is at rest. Therefore, it is assumed that Unit 2, Unit 3 and Unit 4 have a "passive” behaviour on the observed surging of the power plant. Hence draft tube hydro acoustic parameters are applied only for Unit 1 . The stability diagram of the power plant including water 
conduits and turbine units is plotted in Fig. 7 with the set of prototype parameters $a-\chi$ derived by transposition and given in Tab.3.

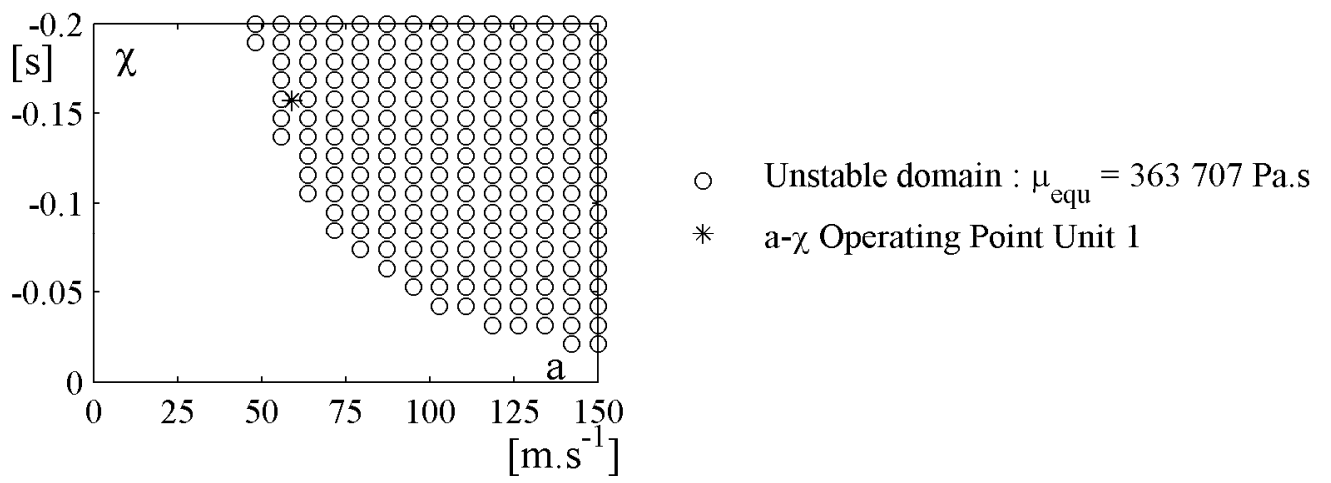

Fig.7 Stability diagram of the power plant

As observed experimentally, the operating point is found to be unstable. The corresponding unstable eigenmode has a frequency value of $f=0.39 \mathrm{~Hz}$, compared to the measurements at $f=0.46 \mathrm{~Hz}$. The spatial shape of the pressure and discharge fluctuations are plotted in Fig. 8, with arbitrary units for the eigenmode amplitudes.
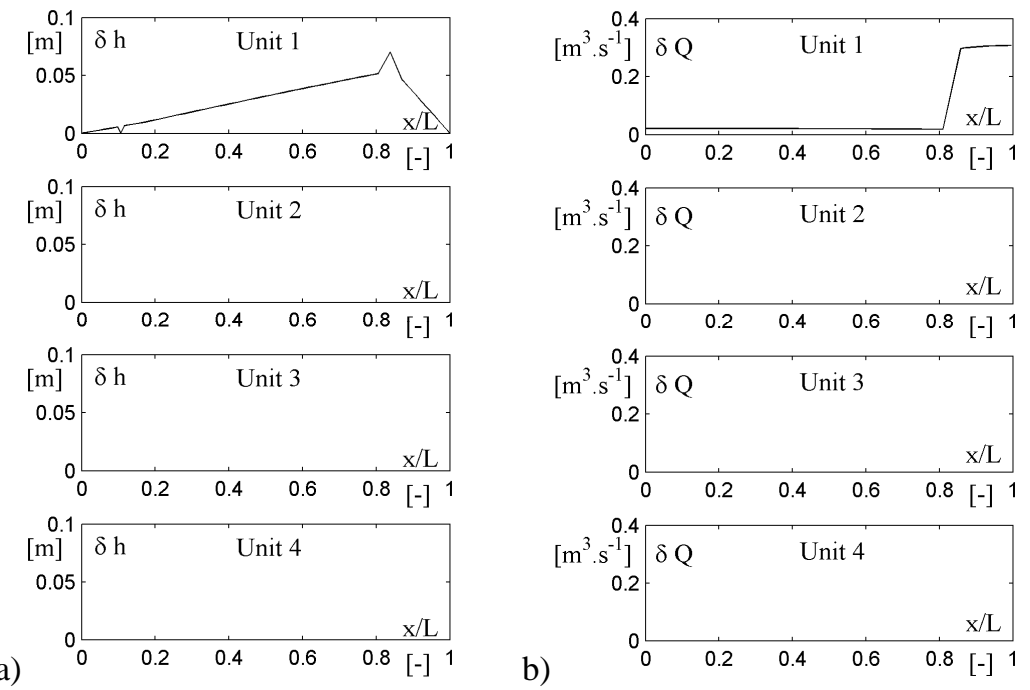

Fig.8 Unstable a) pressure and b) discharge eigenmode.

Each figure is subdivided into four parts which correspond to Unit 1, Unit 2, Unit 3 and Unit 4 branches respectively from the top to the bottom. The $\mathrm{x}$ axis is the length from the upstream to the downstream reservoirs; the surge shaft and the turbine being located respectively at $x / L=0.11$ and $x / L=0.81$. Pressure and discharge fluctuations occur only on Unit 1 as observed experimentally. The standing wave, features large discharge fluctuations between the turbine and the downstream water level due to the vortex rope's large compliance compared to the pipes.

\section{Conclusion}

The frequency of Mica Unit 1 full load instability, determined using the methodology presented in this paper is in general agreement with the prototype measurements. Although the computed instability frequency of $0.39 \mathrm{~Hz}$ is different from the experimental one of $0.46 \mathrm{~Hz}$, the difference is not significant. This difference may be attributed to the following factors. First, the investigated operating point on the reduced scale model does not correspond accurately to the operating point of the surging scenario due to uncertainties of prototype gate openings, power output and Thoma number. Second, the operating conditions on the prototype unit included natural air admission through the shaft into the draft tube and that was not reproduced in the model testing. The air admission changes the hydro acoustic parameters of the draft tube vortex, however, the analysis of this phenomenon was not part of the scope of this study. Finally, the physics simplifications made in the models used in both the SIMSEN and in the CFD numerical simulations also contribute to the difference in results. 


\section{Acknowledgments}

The investigation reported in this paper has been financially and technically supported by BC Hydro which made available the test case for investigation and the prototype measurements for validation. The authors are very grateful to BC Hydro for its involvement and constant support to the project. Finally the staff of the Laboratory for Hydraulic Machines should be acknowledged for its support in the experimental and numerical investigations.

\section{Nomenclature}

\begin{tabular}{|c|c|c|c|}
\hline A & Pipe cross section area $\left[\mathrm{m}^{2}\right]$ & $\tilde{p}$ & Pressure fluctuation $[\mathrm{Pa}]$ \\
\hline $\mathrm{a}$ & Wave speed $\left[\mathrm{m} . \mathrm{s}^{-1}\right]$ & Q & Discharge $\left[\mathrm{m}^{3} \cdot \mathrm{s}^{-1}\right]$ \\
\hline $\mathrm{C}$ & Electrical capacitance $\left[\mathrm{m}^{2}\right]$ & $\mathrm{Q}_{\mathrm{ED}}$ & Discharge factor [-] \\
\hline $\mathrm{C}_{\mathrm{c}}$ & Cavitation compliance $\left[\mathrm{m}^{2}\right]$ & $\delta Q$ & Discharge fluctuation $\left[\mathrm{m}^{3} . \mathrm{s}^{-1}\right]$ \\
\hline$C_{p}$ & Pressure coefficient [-] & $\mathrm{R}$ & Resistance $\left[{\left.\mathrm{s} . \mathrm{m}^{-2}\right]}\right.$ \\
\hline $\mathrm{D}_{\text {ref }}$ & Machine reference diameter [m] & $\mathrm{R}_{\mathrm{ve}}$ & Viscoelastic resistance $\left[\mathrm{s}^{\left.-\mathrm{m}^{-2}\right]}\right.$ \\
\hline $\mathrm{E}$ & Machine specific energy $\left[\mathrm{J} \mathrm{kg}^{-1}\right]$ & $\mathrm{T}_{\mathrm{ED}}$ & Torque factor [-] \\
\hline $\mathrm{f}$ & Frequency [Hz] & $\mathrm{U}_{1}$ & Peripheric velocity $\left[\mathrm{m} . \mathrm{s}^{-1}\right]$ \\
\hline g & Gravity acceleration $\left[\mathrm{m} . \mathrm{s}^{-2}\right]$ & $\mathrm{y}$ & Guide vane opening $\left[^{\circ}\right]$ \\
\hline $\mathrm{h}$ & Piezometric head [m] & $\mathrm{V}_{\mathrm{c}}$ & Cavity volume $\left[\mathrm{m}^{3}\right]$ \\
\hline$\delta$ h & Piezometric head fluctuation [m] & $\rho$ & Density $\left[\mathrm{kg} \cdot \mathrm{m}^{-3}\right]$ \\
\hline l & Length of cone and elbow parts [m] & $\chi$ & Mass flow gain factor $[\mathrm{s}]$ \\
\hline $\mathrm{L}$ & Inductance $\left[\mathrm{s}^{2} \cdot \mathrm{m}^{-2}\right]$ & $\sigma$ & Thoma number [-] \\
\hline $\mathrm{n}$ & Runner frequency [Hz] & $\mu$ & Viscoelastic damping [Pa.s] \\
\hline $\mathrm{N}_{\mathrm{ED}}$ & Speed factor [-] & $\alpha$ & Modal damping [-] \\
\hline
\end{tabular}

\section{References}

[1] T. JACOB, J. PRENAT and MARIA, D., 1988. Comportement dynamique d'une turbine francis à forte charge, comparaisons modèle prototype. La Houille Blanche, Vol 3, p. 293-300.

[2] T. JACOB, J.E. PRÉNAT, G. VULLIOUD and ARAGUAS, B.L., 1992. Surging of 140MW Francis turbines at high load, analysis and solution. IAHR Symposium on Hydraulic Machinery and Systems, Sao Paolo.

[3] C. BRENNEN and ACOSTA, A.J., 1976. The dynamic transfer function for a cavitating inducer. Journal of fluids engineering ASME, Vol 98, p. 182-191.

[4] C. BRENNEN, 1978. Bubbly flow model for the dynamic characteristics of cavitating pumps. Journal of Fluid Mechanics, Vol 89, p. 223-240.

[5] J. KOUTNIK and PULPITEL, L., 1996. Modelling of the Francis turbine full load surge. IAHR Symposium on Hydraulic Machinery and Systems, Lausanne.

[6] Y. TSUJIMOTO, K. KAMIJO and YOSHIDA, Y., 1993. Theorical analysis of rotating cavitation in inducers. Journal of fluids engineering, ASME, Vol 115, p. 135-141.

[7] M.E. DUTTWEILER and BRENNEN, C., 2002. Surge instability on a cavitating propeller. Journal of fluids Mechanics, Vol 458, p. 133-152.

[8] S. WATANABE and BRENNEN, C., 2003. Dynamics of a cavitating propeller in a water tunnel. Journal of fluids engineering, ASME, Vol 125, p. 283-292.

[9] C. CHEN, C. NICOLET, K. YONEZAWA, M. FARHAT, F. AVELLAN and TSUJIMOTO, Y., 2008. One dimensional analysis of full load draft tube suge. Journal of Fluids Engineering, Vol 130, p. 041106-1; 041106-6.

[10] F. FLEMMING, J. FOUST, J. KOUTNIK and FISHER, R.K., 2008. Overload surge investigation using CFD data. IAHR Symposium on Hydraulic Machinery and Systems, Foz Do Iguassu, Brazil.

[11] J. KOUTNIK, C. NICOLET, G.A. SCHOHL and AVELLAN, F., 2006. Overload surge event in a pumped storage power plant. IAHR Symposium on Hydraulic Machinery and Systems, Yokohama.

[12] S. ALligne, C. NICOLET, N. RUCHONNET, V. HASMATUCHI, P. MARUZEWSKI and AVELLAN, F., 2009. Numerical Simulation of nonlinear self oscillations of a full load vortex rope. 3rd IAHR International Meeting of the workgroup on cavitation and dynamic problems in Hydraulic Machinery and Systems, Brno, Czech Republic.

[13] IEC 60193 Standard -1999. Hydraulic Turbines, Storage Pumps and Pump-Turbines Model Acceptance Tests.

[14] J.P. FRANC, F. AVELLAN, et al., 1995. La cavitation.

[15] PEZZINGA, G., 2003. Second viscosity in transient cavitating pipe flows. IAHR Journal of Hydraulic research, Vol 6, p. 656-665.

[16] MENTER, F.R., 1994. Two-Equation Eddy-Viscosity Turbulence Models for Engineering Applications. AIAA Journal, Vol 32, p. 1598-1605.

[17] S.ALLIGNÉ, C. NICOLET, P. ALLENBACH, B. KAWKABANI, J.J. SIMOND and AVELLAN., F., 2008. Influence of the vortex rope location of a Francis Turbine on the hydraulic system stability. IAHR Symposium on Hydraulic Machinery and Systems, Brazil. 